Am. J. Trop. Med. Hyg., 77(Suppl 6), 2007, pp. 128-132

Copyright $(2007$ by The American Society of Tropical Medicine and Hygiene

\title{
The Cost-Effectiveness of Parasitologic Diagnosis for Malaria-Suspected Patients in an Era of Combination Therapy
}

\author{
Yoel Lubell,* Hugh Reyburn, Hilda Mbakilwa, Rose Mwangi, Kini Chonya, Christopher J. M. Whitty, and Anne Mills \\ Department of Public Health and Policy, London School of Hygiene and Tropical Medicine, London, United Kingdom; Department \\ of Infectious and Tropical Disease, London School of Hygiene and Tropical Medicine, London, United Kingdom; Joint Malaria
} Programme, Kilimanjaro Christian Medical Centre, Moshi, Tanzania

\begin{abstract}
The introduction of artemisinin-based combination therapy in sub-Saharan Africa has prompted calls for increased use of parasitologic diagnosis for malaria. We evaluated the cost-effectiveness of rapid diagnostic tests (RDTs) in comparison to microscopy in guiding treatment of non-severe febrile illness at varying levels of malaria endemicity using data on test accuracy and costs collected as part of a Tanzanian trial. If prescribers complied with current guidelines, microscopy would give rise to lower average costs per patient correctly treated than RDTs in areas of both high and low transmission. RDT introduction would result in an additional $2.3 \%$ and $9.4 \%$ of patients correctly treated, at an incremental cost of $\$ 25$ and $\$ 7$ in the low and high transmission settings, respectively. Cost-effectiveness would be worse if prescribers do not comply with test results. The cost of this additional benefit may be higher than many countries can afford without external assistance or lower RDT prices.
\end{abstract}

\section{INTRODUCTION}

In response to the growth of resistance to standard antimalarials, artemisinin-based combination therapy (ACT) is being introduced as a first-line drug in much of sub-Saharan Africa (SSA). ${ }^{1}$ Its cost and the increasing recognition that presumptive treatment leads to considerable drug expenditure on inappropriate treatment of patients free of parasitemia have led to increased interest in parasitologic diagnosis. WHO and Tanzanian national guidelines now both recommend testing before treatment of patients over the age of 5 years, ${ }^{2,3}$ and WHO guidelines extend this recommendation to younger children in low transmission settings.

In smaller health facilities in Africa, the only currently realistic method of parasitologic diagnosis is through the use of rapid diagnostic tests (RDTs). In larger facilities, where microscopic diagnosis is used, the results are frequently unreliable. This has led the Tanzanian National Malaria Control Program to consider the use of RDTs for all routine outpatient malaria diagnosis and to reserve the use of microscopy to cases where a measure of parasite species or density is needed or where treatment has been recently taken.

RDTs are a promising tool to target antimalarial treatment because they require minimal infrastructure and simple training. RDTs have been shown to have sensitivity and specificities of $>90 \%$ in field conditions, a level rarely sustained for routine microscopy in resource-constrained African countries. ${ }^{4-6}$ Additionally, because RDTs are potentially able to provide rapid results that are directly visible to both prescriber and patient, they have potential operational advantages over microscopy. However, the cost implications of their deployment relative to microscopy have not been extensively studied. These may be considerable, and if so, will be an influential factor in decisions whether to deploy them in health facilities where microscopy already exists. ${ }^{2,7}$

\section{MATERIALS AND METHODS}

In July 2005, a randomized controlled trial was conducted in three public hospitals in northeast Tanzania serving areas

* Address correspondence to Yoel Lubell, London School of Hygiene and Tropical Medicine, Keppel Street, London WC1E 7HT, UK. E-mail: yoel.lubell@lshtm.ac.uk where the transmission of malaria has previously been characterized as very low, low, and high (parasite prevalence in children younger than 5 years of age of $2 \%, 5 \%$, and $61 \%$, respectively). ${ }^{8}$ Patients $(N=2,416)$ for whom the clinician had requested a parasitologic test for malaria were randomized to diagnosis using routine microscopy or an RDT for the detection of Pf Histidine Rich Protein 2 antigen (ParacheckPf, Orchid Biomedical Systems, Goa, India). In both arms, reference slides were taken and later double-read according to research methods. ${ }^{9}$ Data on treatments given were recorded.

The aim of the trial was 2-fold: to assess the accuracy of RDTs compared with routine microscopy, and to examine the effect of test results on clinician behavior. This paper focuses on the former, using trial data on the tests' accuracy and cost, combined with ACT costs, to analyze the cost-effectiveness of the use of the two tests to guide treatment in an operational setting, where test performance is judged against the gold standard of a double-read research slide. The cost and effectiveness data were applied to parasite prevalence rates observed in the high and low transmission settings to compare the relative efficiency of each diagnostic approach in the two settings. The results of the low and medium-low transmission settings were combined in the analysis.

The analysis simulated scenarios where all patients, and then only patients older than 5 years of age, were treated according to parasitologic confirmation, representing the two most common diagnostic algorithms recommended in malaria-endemic countries. The main implications of including the youngest age group are that younger patients require smaller dosages, therefore incurring lower direct treatment costs, and that parasite prevalence among young children is higher, particularly in high transmission settings.

Test sensitivity and specificity were determined by comparing pooled data on routine microscopy and on RDT results from all three trial sites to reference slide results.

Costs were obtained from data collected in the low and high prevalence settings, supplemented where necessary by data from the literature. The costing perspective was that of the provider. Costs were collected in Tanzanian Shillings of 2005 and converted to US dollars ( $\$ 1=1,167$ Tzs for 2005).

Slide costing combined micro-costing (noting all resources used at the point of delivery and using an ingredients ap- 
proach to calculate their total economic cost) with step-down costing of hospital expenditure to estimate indirect laboratory costs. These costs were apportioned to the various laboratory tests according to their proportional activity. Data for stepdown costing were obtained from hospital accounts and a recent independent evaluation of all hospital assets.

The cost of RDTs was obtained directly from the manufacturer and included shipment costs plus $10 \%$ for local transport and storage. The time needed for administration of the tests was assumed to be equivalent to the preparation of a blood slide.

The cost of ACT was estimated at $\$ 2.4$ for an adult course of artemether-lumefantrine, the current price negotiated between the World Health Organization and Novartis, the manufacturer, ${ }^{2}$ and adjusted for patient age. These costs were used in the analysis in place of costs of currently available antimalarials to simulate the switch to artemether-lumefantrine (Coartem, Novartis Pharma, Basel, Switzerland) in Tanzania as the first-line drug for the treatment of uncomplicated malaria.

The cost of treatment of patients diagnosed as malaria negative was estimated from data on the treatment cost of trial patients who received an antibiotic but not an antimalarial. The geometric mean was used for these because they were highly skewed to the right.

The measure of effectiveness for this analysis was defined as the proportion of patients correctly treated, in the context of a strategy of parasitologic confirmation, assuming that patients with a positive test received an antimalarial, and patients that tested negative received an antibiotic. These two measures were summed so that the provision of an antimalarial to a true positive and the provision of alternative treatment to true negatives were both included.

To evaluate the cost-effectiveness of the two diagnostic strategies more broadly, the results were modeled across a range of prevalence levels, assuming that all age groups were tested and treated according to test results as defined above. Two-way sensitivity analyses were carried out on malaria prevalence and the cost of ACT, varying the latter to the estimated cost of $\$ 1$ for a cheaper combination and to a lowest estimate for a scenario of high subsidies reducing their cost to that of currently used antimalarials. Similarly, the cost of the RDT was varied to assess the impact of a drop in price (to an estimated minimum of 20 cents), which might result from their larger-scale production.

In addition to an intervention's cost-effectiveness, policy makers need information on affordability. Thus, the total costs were estimated for the management of 1,000 malariasuspected patients, using either microscopy or RDTs for diagnosis before treatment with ACT.

\section{RESULTS}

Microscopy costs. The cost per slide was $\$ 0.26$. Labor was the largest cost component, although staff took an average of $<1.5$ minutes before declaring a slide negative or providing a positive result along with a parasitemia count.

Rapid diagnostic test costs. RDT costs were $\$ 0.81$, the most significant component being the test itself $(\$ 0.60)$.

Treatment costs. Geometric mean cost for treatment of patients diagnosed as not having a malarial illness was $\$ 0.42$ compared with $\$ 2.40$ for an adult course of Coartem.

Observed diagnostic accuracy and prevalence. Test specificity was comparably high for both tests-95\% for RDT and 93\% for microscopy-but RDTs were substantially more sensitive $(93 \%)$ than routine microscopy $(71 \%)$ in this setting.

Data on test accuracy and prevalence were combined to estimate the percentage of patients correctly treated. Table 1 summarizes the results for a strategy where all patients are tested before treatment and also summarizes the results for treating only patients older than 5 years of age by parasitologic confirmation. The difference in the proportion of patients correctly diagnosed by each method in areas of low transmission is smaller, despite the lower sensitivity of slides, a result of the lower likelihood of parasitemia.

Cost-effectiveness of RDTs and microscopy. Estimates of cost-effectiveness per patient correctly treated were obtained by summing diagnosis and treatment costs. These were divided by the proportion of patients correctly treated to determine the average cost per patient correctly treated. Incremental costs were extrapolated from this in the standard manner. ${ }^{10}$ The incremental cost represents the cost per additional patient correctly treated by switching to the use of RDTs.

Low transmission setting. Under observed levels of slide accuracy, if provision of ACT was reliant on a positive blood slide and all patients were tested before treatment, the percentage of patients correctly treated would be $92.7 \%$, at an average cost per patient of $\$ 0.7$. Replacing microscopy by RDTs would raise the percentage of patients correctly treated to $95 \%$, at an average cost of $\$ 1.3$ per patient correctly treated. The incremental cost for each additional patient treated correctly would be $\$ 25.2$ in comparison to the use of microscopy. If only patients older than 5 years of age were tested before treatment, the results would vary only slightly because of the small difference in prevalence by age group.

High transmission setting. In the high transmission setting, prevalence rates were considerably higher, which in conjunction with the lower sensitivity obtained with microscopy would result in $88.6 \%$ of patients being correctly treated at an average cost of $\$ 1.1$. With the use of RDTs, the percentage of

TABLE 1

Diagnostic accuracy of microscopy and RDTs according to the reference slide results in two transmission settings

\begin{tabular}{|c|c|c|c|c|c|c|c|c|}
\hline \multirow[b]{3}{*}{ Transmission } & \multicolumn{4}{|c|}{ All patients are tested } & \multicolumn{4}{|c|}{ Only patients $\geq 5$ years of age are tested } \\
\hline & \multicolumn{2}{|c|}{ Low $($ prev $=1.3 \%)$} & \multicolumn{2}{|c|}{ High $($ prev $=38.1 \%)$} & \multicolumn{2}{|c|}{ Low $($ prev $=1.55 \%)$} & \multicolumn{2}{|c|}{ High $($ prev $=20.8 \%)$} \\
\hline & RDT & Slide & RDT & Slide & RDT & Slide & RDT & Slide \\
\hline Missed case of malaria & $0.1 \%$ & $0.4 \%$ & $2.6 \%$ & $11 \%$ & $0.1 \%$ & $0.4 \%$ & $1.5 \%$ & $6.1 \%$ \\
\hline Wrongly diagnosed malaria & $4.9 \%$ & $6.9 \%$ & $3.1 \%$ & $4.3 \%$ & $4.9 \%$ & $6.9 \%$ & $4 \%$ & $5.5 \%$ \\
\hline Correct diagnosis of malaria & $1.2 \%$ & $0.9 \%$ & $35.4 \%$ & $27 \%$ & $1.4 \%$ & $1.1 \%$ & $19.5 \%$ & $14.5 \%$ \\
\hline Correct diagnosis of non-malaria & $93.8 \%$ & $91.8 \%$ & $58.9 \%$ & $57.7 \%$ & $93.6 \%$ & $91.6 \%$ & $75 \%$ & $73.5 \%$ \\
\hline Patients correctly diagnosed & $95 \%$ & $92.7 \%$ & $94.2 \%$ & $84.6 \%$ & $95 \%$ & $92.7 \%$ & $94.5 \%$ & $88.4 \%$ \\
\hline
\end{tabular}




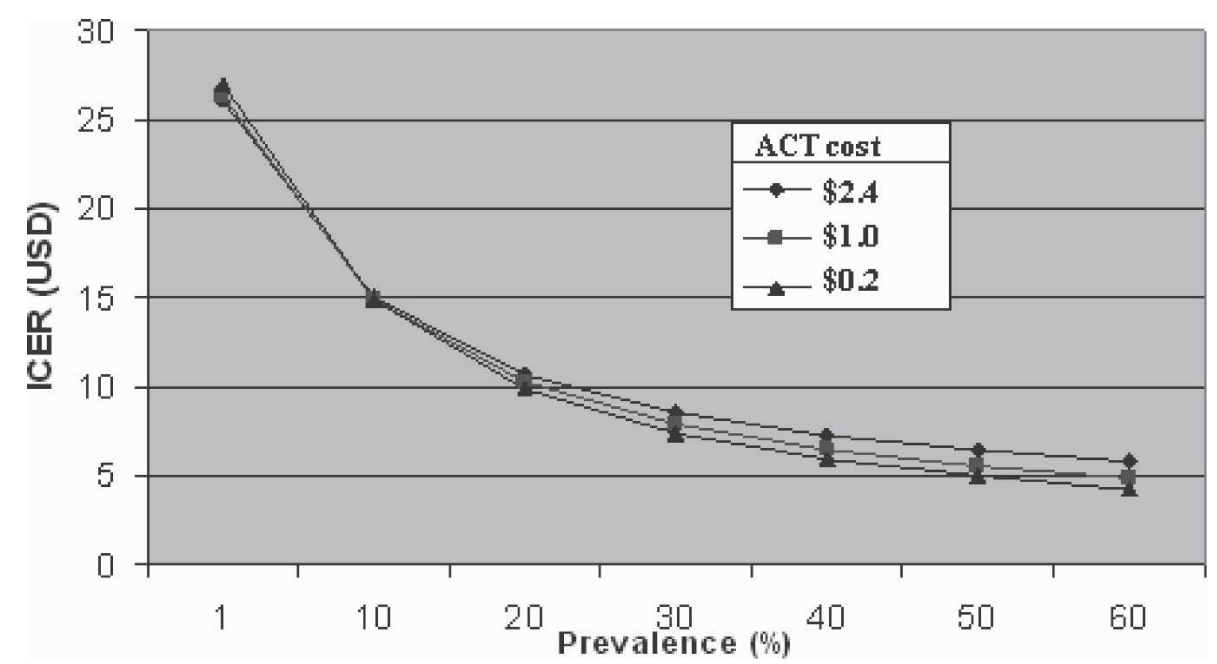

FIGURE 1. ICER for RDTs compared with microscopy by prevalence, with different price levels for ACT.

patients correctly treated would be $94.2 \%$, with an average cost of $\$ 1.7$. Because of the larger difference in effectiveness, the incremental cost would drop to $\$ 7.0$ for each additional patient correctly treated. If parasitic confirmation was required only for patients older than 5 years of age, their lower prevalence $(21 \%)$ would result in a slightly higher incremental cost-effectiveness ratio (ICER) of $\$ 10.1$.

Differences in patient costs. In the trial, both tests were administered to patients free of charge. The only significant difference between the use of RDTs and microscopy was in terms of total duration of the visit. Time from exiting first to exiting second consultations was found to be lower by $\sim 50$ minutes among patients randomized to the RDT group.

Sensitivity analyses. Figure 1 plots the incremental costeffectiveness of RDTs compared with microscopy against a range of prevalences, showing that they become increasingly cost-effective as prevalence increased. Figure 1 also shows the effect of different ACT costs on the ICER. These include a lower ACT cost to simulate the introduction of a cheaper drug (such as dihydroartemisinin-piperaquine at $\sim 1$ USD per adult dose) and a much lower ACT cost to simulate a subsidy that would reduce the cost to the consumer to that of current antimalarials. As Figure 1 indicates, this had little impact on the incremental cost, which was almost identical for all three ACT prices.

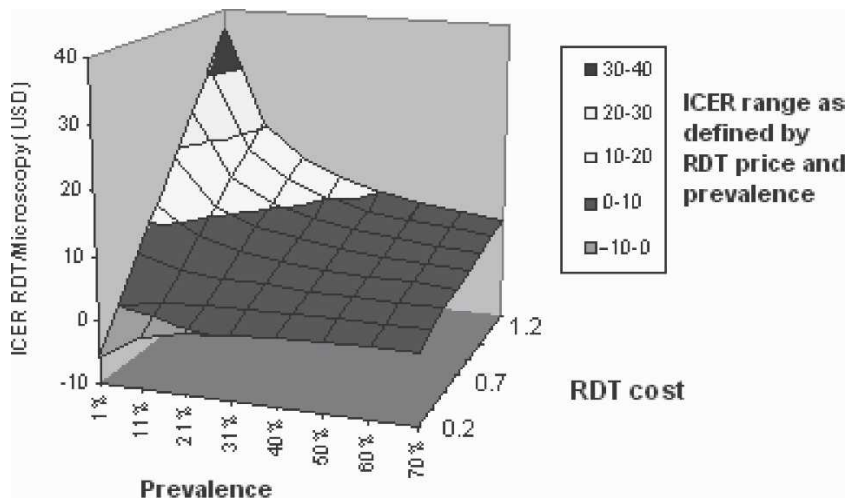

FIGURE 2. The ICER in relation to prevalence and RDT cost.
The ICER was highly sensitive to change in the price of RDTs, particularly where prevalence was low. As Figure 2 shows, should the price drop $<29$ cents, RDTs could become cost saving. At high prevalence, the ICER was far less sensitive, although always less than $\$ 10$ per patient correctly treated.

Affordability. The total budget needed to treat 1,000 malaria-suspected patients of all ages (a similar age structure to that documented in the trial) by parasitologic confirmation was consistently lower for microscopy in both transmission settings, ranging from $\$ 932$ to $\$ 1,260$, as opposed to $\$ 1,507$ to $\$ 1,633$ for RDTs (Figure 3). The difference between the two is explained both by the higher cost of RDTs and by their higher sensitivity with consequent higher use of ACTs.

Replacement of microscopy by RDTs would thus increase costs by $61 \%$ and $30 \%$ at low and high prevalences, respectively.

\section{DISCUSSION}

On the assumption that the introduction of more expensive antimalarials will stimulate the increased use of parasitologic diagnosis, our analysis compared the use of RDTs to microscopy in health facilities where the latter is available. We assume full compliance of clinicians with test results because the

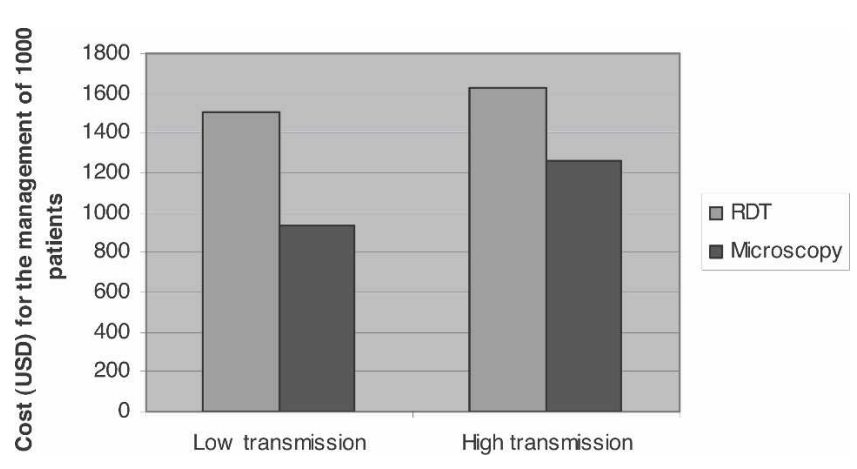

FIgURE 3. Total costs for management of 1,000 patients with the use of either RDTs or microscopy. 
efficient use of parasitologic diagnosis is contingent on compliance, as shown recently by Zerovac and others ${ }^{11}$ in their evaluation of the financial implications of compliance with microscopy results before treatment with artemetherlumefantrine for febrile patients in Kenya.

We showed that, in both high and low transmission settings, the use of microscopy results in lower average costs per patient correctly treated than the use of RDTs. However, the slide results in this study were both less sensitive and specific than RDT results. The incremental cost for each additional patient correctly treated because of the higher accuracy of RDTs ranged from $\$ 7$ to $\$ 26$, depending on transmission intensity. Variations in ACT prices had little effect on results, but they were very sensitive to reductions in the RDT price, especially in the low transmission setting.

A multitude of studies have been carried out on the accuracy of RDTs in comparison to microscopy, but only three studies were identified that compared their costeffectiveness, ${ }^{12-16}$ and only one of these incorporated treatment costs. This study was conducted in Southeast Asia, with significantly different epidemiologic and economic circumstances. ${ }^{12}$ The study here goes beyond these studies. It evaluates the efficiency of RDTs within hospital settings where microscopy is readily available, and it incorporates ACT costs in an SSA setting. In terms of the interventions' effectiveness, our study includes the benefit of focusing on diagnosis not just for malaria patients but also for those found to be parasite negative.

In settings where microscopy is not a feasible option, the cost-effectiveness of RDTs needs to be compared with that of clinical diagnosis and presumptive treatment, the results of which may differ substantially from our findings. Although this was not the topic of our analysis, this will be a relevant issue in many SSA locations.

Although data were drawn from a specific setting in Tanzania, there is no reason to believe that this setting is atypical. The unit cost of blood slides, at $\$ 0.26$, was comparable to those calculated in previous studies, which ranged from $\$ 0.1$ to $\$ 0.58 .{ }^{11,14,17,18}$ Results from studies that have evaluated the accuracy of microscopy were either comparable to those found in the trial ${ }^{12,19}$ or were somewhat worse. ${ }^{11,20}$ Where the latter is the case, RDTs will seem somewhat more costeffective than findings here suggest.

The study has several limitations. With respect to costs, only provider treatment and diagnosis costs were included and not those for readmission of patients wrongly diagnosed/ treated or indirect costs such as productivity losses. Had these been included, the cost-effectiveness of both tests, and of RDTs in particular, is likely to have been greater. Patients diagnosed with an RDT had a shorter wait, which if allowed for would slightly reduce the additional cost of RDTs compared with microscopy. Any inherent value to providers and patients of speedier diagnosis was not allowed for.

Slide costs were treated as fixed, although in practice, they will vary considerably with respect to volume. Because the costs for maintaining microscopy facilities and technicians remains fixed, lowering the output increases the average unit cost. ${ }^{14,17}$ This implies that, in peripheral dispensaries where the volume is lower than that observed in the trial hospitals, RDTs will be increasingly cost-effective.

"Patients correctly treated" was defined as provision of antimalarials to test positives; for patients who were test nega- tive, there was no such convenient definition of correct treatment without further information on the exact nature of other febrile illnesses. In the absence of better data, it was assumed that the treatment cost of test negatives was represented by that of trial patients who received an antibiotic and not an antimalarial. In reality, a more diverse range of treatments may be given to test negatives.

The huge burden of malaria in Africa means that even small differences in the cost of treatment or diagnosis can have large economic effects. In the public sector in Tanzania in 2004, > 11 million treatments for malaria were prescribed; the extent of slide testing in these patients is not routinely reported, but several studies have now documented that a high proportion of treatments for malaria are in patients who are either not tested or who have been tested but have a negative result.

The much higher drug costs that are resulting from the deployment of ACT is stimulating debate on the role of parasitologic diagnosis. We showed that the choice between RDTs and microscopy is not straightforward, because RDTs were found to be not just more accurate but also more costly. In the context of SSA health budgets, the additional cost of up to $\$ 26$ for the benefit of correctly treating a patient is not insignificant, suggesting that both external assistance for increasing the volume of diagnosis and finding ways of reducing the cost of RDTs are important. Moreover, regardless of which diagnostic test is used, attention must be focused on encouraging clinician compliance with test results, because the lower this is, the worse the cost-effectiveness of diagnosis and treatment will be.

Cost-effectiveness studies such as this should ideally include extrapolation to final health outcomes. This is especially important to capture adequately the benefits of testing for patients with non-malaria febrile illnesses. Lack of information on such illnesses led us to evaluate the diagnostic tests in terms of patients correctly treated. Improving the evidence base to permit a complete cost-effectiveness analysis is a research priority.

Received August 21, 2006. Accepted for publication February 23, 2007.

Acknowledgments: The authors thank Catherine Goodman, Chris Drakely, Jack Dowie, John Cairns, and Gesine Meyer-Rath for their advice in designing the study and critical comments on the manuscript. This study was conducted within the Joint Malaria Programme, a collaboration between the National Institute for Medical Research in Tanzania, Kilimanjaro Christian Medical Centre, London School of Hygiene and Tropical Medicine, and the University of Copenhagen. Alan Minja, Kereja Mlay, and Rajabu Malahiyo were key hospital staff in each site. Anna Mtei, Emmanuel Mwakasungula, Lilian Ngowi, Boniface Njau, Yustina Mushi, Happiness Manaso, Mary Urio, and Nico Funga collected the outpatient data. Magdalena Massawe, James Kalabashanga, and Hatibu Athumani read research blood films. Prof Raimos Olomi contributed to the study design. We thank the patients and staff in study sites for their support and participation.

Financial support: The trial was sponsored by the Gates Malaria Programme with funds from the Bill \& Melinda Gates Foundation, as part of the Joint Malaria Programme of NE Tanzania.

Authors' addresses: Yoel Lubell, Chris Whitty, and Anne Mills, London School of Hygiene and Tropical Medicine, Keppel Street, London WC1E 7HT, UK, Fax: 44-0-20-7637-5391. Hugh Reyburn, Hilda Mbakilwa, Rose Mwangi, and Kini Chonya, Joint Malaria Programme, PO Box 2228, KCMC, Moshi, Tanzania, Telephone: 027275-3714, Fax: 02-7275-3982. 


\section{REFERENCES}

1. Coleman PG, Morel C, Shillcutt S, Goodman C, Mills AJ, 2004. A threshold analysis of the cost-effectiveness of artemisininbased combination therapies in sub-saharan Africa. Am J Trop Med Hyg 71: 196-204.

2. WHO, 2006. Guidelines for the Treatment of Malaria. Geneva: The World Health Organization.

3. NMCP, 2005. Diagnosis and Treatment of Malaria Orientation Guide for District Trainers. Tanzania: Ministry of Health.

4. Moody A, 2002. Rapid diagnostic tests for malaria parasites. Clin Microbiol Rev 15: 66-78.

5. Murray CK, Bell D, Gasser RA, Wongsrichanalai C, 2003. Rapid diagnostic testing for malaria. Trop Med Int Health 8: 876-883.

6. WHO, 2004. The Use of Malaria Rapid Diagnostic Tests. Geneva: The World Health Organization.

7. Nosten F, Ashley E, 2004. The detection and treatment of Plasmodium falciparum malaria: time for change. J Postgrad Med 50: 35-39.

8. Drakeley CJ, Carneiro I, Reyburn H, Malima R, Lusingu JP, Cox J, Theander TG, Nkya WM, Lemnge MM, Riley EM, 2005. Altitude-dependent and independent variations in Plasmodium falciparum prevalence in northeastern Tanzania. $J$ Infect Dis 191: 1589-1598.

9. Reyburn H, Mbakilwa H, Mwangi R, Mwerinde O, Olomi R, Drakeley D, Whitty CJ, 2007. Rapid diagnostic tests compared with malaria microscopy for guiding outpatient treatment of febrile illness in Tanzania: a randomised trial. BMJ 334: 403.

10. Drummond MF, O'Brien B, Stoddart GL, Torrance GW, 1997. Methods for the Economic Evaluation of Health Care Programmes. Oxford: Oxford University Press.

11. Zurovac D, Larson BA, Akhwale W, Snow RW, 2006. The financial and clinical implications of adult malaria diagnosis using microscopy in Kenya. Trop Med Int Health 11: 1185-1194.

12. Bualombai P, Prajakwong S, Aussawatheerakul N, Congpoung K, Sudathip S, Thimasarn K, Sirichaisinthop J, Indaratna K, Kidson C, Srisuphanand M, 2003. Determining cost-effective- ness and cost component of three malaria diagnostic models being used in remote non-microscope areas. Southeast Asian J Trop Med Public Health 34: 322-333.

13. Fernando SD, Karunaweera ND, Fernando WP, Attanayake N, Wickremasinghe AR, 2004. A cost analysis of the use of the rapid, whole-blood, immunochromatographic P.f/P.v assay for the diagnosis of Plasmodium vivax malaria in a rural area of Sri Lanka. Ann Trop Med Parasitol 98: 5-13.

14. Goodman C, Coleman PG, Mills A, 2000. Economic Analysis of Malaria Control in Sub-Saharan Africa. Geneva: Global Forum for Health Research.

15. Pang LW, Piovesan-Alves F, 2001. Economic advantage of a community-based malaria management program in the Brazilian Amazon. Am J Trop Med Hyg 65: 883-886.

16. Rolland E, Checchi F, Pinoges L, Balkan S, Guthmann JP, Guerin PJ, 2006. Operational response to malaria epidemics: are rapid diagnostic tests cost-effective? Trop Med Int Health 11: $398-408$.

17. Jonkman A, Chibwe RA, Khoromana CO, Liabunya UL, Chaponda ME, Kandiero GE, Molyneux ME, Taylor TE, 1995. Cost-saving through microscopy-based versus presumptive diagnosis of malaria in adult outpatients in Malawi. Bull World Health Organ 73: 223-227.

18. Agnamey P, Brasseur P, Cisse M, Gaye O, Dumoulin J, Rigal J, Taylor WR, Olliaro P, 2005. Economic evaluation of a policy change from single-agent treatment for suspected malaria to artesunate-amodiaquine for microscopically confirmed uncomplicated falciparum malaria in the Oussouye District of south-western Senegal. Trop Med Int Health 10: 926-933.

19. Stow NW, Torrens JK, Walker J, 1999. An assessment of the accuracy of clinical diagnosis, local microscopy and a rapid immunochromatographic card test in comparison with expert microscopy in the diagnosis of malaria in rural Kenya. Trans $R$ Soc Trop Med Hyg 93: 519-520.

20. Reyburn H, Ruanda J, Mwerinde O, Drakeley C, 2006. The contribution of microscopy to targeting antimalarial treatment in a low transmission area of Tanzania. Malar J 5: 4. 